\title{
Chaotic Dynamics-Based Analysis of Broadband Piezoelectric Vibration Energy Harvesting Enhanced by Using Nonlinearity
}

\author{
Zhongsheng Chen, Bin Guo, Congcong Cheng, Hongwu Shi, and Yongmin Yang
}

Science and Technology on Integrated Logistics Support Laboratory, National University of Defense Technology, Changsha 410073, China

Correspondence should be addressed to Zhongsheng Chen; czs_study@sina.com

Received 31 August 2015; Revised 22 October 2015; Accepted 27 October 2015

Academic Editor: Carlo Trigona

Copyright (C) 2016 Zhongsheng Chen et al. This is an open access article distributed under the Creative Commons Attribution License, which permits unrestricted use, distribution, and reproduction in any medium, provided the original work is properly cited.

\begin{abstract}
Nonlinear magnetic forces are always used to enlarge resonant bandwidth of vibration energy harvesting systems with piezoelectric cantilever beams. However, how to determine properly the distance between two magnets is one of the key engineering problems. In this paper, the Melnikov theory is introduced to overcome it. Firstly, the Melnikov state-space model of the nonlinear piezoelectric vibration energy harvesting (PVEH) system is built. Based on it, chaotic dynamics mechanisms of achieving broadband PVEH by nonlinearity are exposed by potential function of the unperturbed nonlinear PVEH system. Then the corresponding Melnikov function of the nonlinear PVEH system is defined, based on which two Melnikov necessary conditions of determining the distance are obtained. Finally, numerical simulations are done to testify the theoretic results. The results demonstrate that the distance is closely related to the excitation amplitude and frequency once geometric and material parameters are fixed. Under a singlefrequency excitation, the nonlinear PVEH system can generate a periodic vibration around a stable point, a large-amplitude vibration around two stable points, or a chaotic vibration. The proposed method is very valuable for optimally designing and utilizing nonlinear broadband PVEH devices in engineering applications.
\end{abstract}

\section{Introduction}

Nowadays, Internet of Things (IoTs) is a research and application hot spot, which can allow objects to be sensed and controlled remotely across existing network infrastructure. A great number of embedded or wireless sensor nodes (WSNs) are distributed in wide and remote areas, such as environmental monitoring, bridge health monitoring, and smart housing. However, there is an urgent need to provide long-term and reliable electrical power for those WSNs. Traditional solutions are to utilize batteries for main sources of WSNs. As we all know that batteries have the drawback of limited life span, they need to be replaced regularly. However, it is often costly and difficult to replace so many batteries, in particular in highly dangerous or unreachable areas. Hence, it is essential for WSNs to have autonomous and long-life power sources. Fortunately, advances in low power design open the possibility of harvesting ambient vibration energy to power WSNs, which is called vibration energy harvesting including electrostatic [1], electromagnetic [2], and piezoelectric mechanisms [3]. In particular, piezoelectric mechanisms are of particular interest due to their high energy densities, no electromagnetic inference, and integration potential, which is always called piezoelectric vibration energy harvesting (PVEH).

In early studies, linear PVEH devices composed of a piezoelectric cantilever beam and a mass attached to the free end are widely investigated. As the piezoelectric cantilever beam oscillates with the host base, the induced mechanical strain produces charges across two electrodes, which can be captured and stored to generate an AC voltage. According to existing conclusions [4], a linear PVEH system can produce the maximum power only when the excitation frequency is equal to its resonant frequency. Otherwise, its harvesting efficiency will greatly decrease once the excitation frequency deviates a little from the resonant frequency. On the other hand, resonant frequency of a PVEH device is closely related to its geometries, so its volume often has to be large in order to reduce the resonant frequency. Consequently, there 
are many limitations of linear PVEH devices in engineering applications, such as self-powered embedded wireless sensors. The first reason is that frequency spectrum of ambient vibration is usually broad and time-varying, instead of being sharply peaked at some frequency. The second reason is that frequency spectrums of many ambient vibrations are particularly rich in low-frequency regions, so it is very difficult to build small-scale, low-frequency resonant PVEH systems.

In order to overcome the above limitations, nonlinear PVEH systems have been proposed for ten years. Gammaitoni et al. [5] firstly discussed vibration to electricity energy conversion mechanisms by using nonlinear stochastic dynamics. Chen et al. [6] built a general nonlinear PVEH model by combining nonlinear stiffness, nonlinear damping, and nonlinear piezoelectric coupling coefficient simultaneously. Based on it, the effects of different nonlinear parameters on the PVEH system were investigated. By this way, bandwidth of useful frequencies has the potential to be extended. Up to now, most works on nonlinear PVEH can be divided into two classes. The first class is based on Duffing-like equations [5, 7-9]. Under this case, a Duffing-like model is build based on nonlinear dynamics and then analyzed by purely numerical simulations. It can be used to expose the effects of nonlinearity, but the numerical model has little physical meaning and thus is unable to guide practical designing. The second class is based on theoretical analysis and experimental testing [10-12]. For a given PVEH structure, theoretical analysis is done to demonstrate its nonlinearity and experiments are done to validate its broadband characteristics. Among all studies, it is the most easy and common way to construct nonlinear PVEH systems by using magnetic forces [13-17]. According to existing studies, the distance between two magnets is a key geometric parameter once other geometric and material parameters are fixed. It should be determined carefully to construct an expected nonlinear PVEH system. However, how to easily determine the distance between two magnets is still an urgent problem in engineering applications. Otherwise, many trials have to be done, which is much more inconvenient and costly. Thus it is necessary to propose a quantitative criterion of determining the distance.

The Melnikov theory provides a unified framework for studying transitions and chaos in a wide class of deterministic and stochastic nonlinear planar dynamic systems with restoring forces. It can be used to find necessary conditions for homoclinic bifurcations to occur in multiwell systems [18]. This is the same case as nonlinear PVEH systems where restoring forces come from electromechanical coupling force and nonlinear magnetic force. The Melnikov criterion helps in an indirect way in ascertaining the values of different parameters for bifurcations. Stanton et al. [19] numerically studied a Melnikov-based method to characterize the dynamics of the bistable piezoelectric inertial generator, but there is no obvious physical meaning. The goal of this paper is to build necessary conditions of determining a proper distance between two magnets based on the Melnikov method. The left contents are organized as follows: state-space model of one nonlinear PVEH system with nonlinear magnetic force is built in Section 2. In Section 3, a perturbed state-space representation of the nonlinear PVEH system is presented,

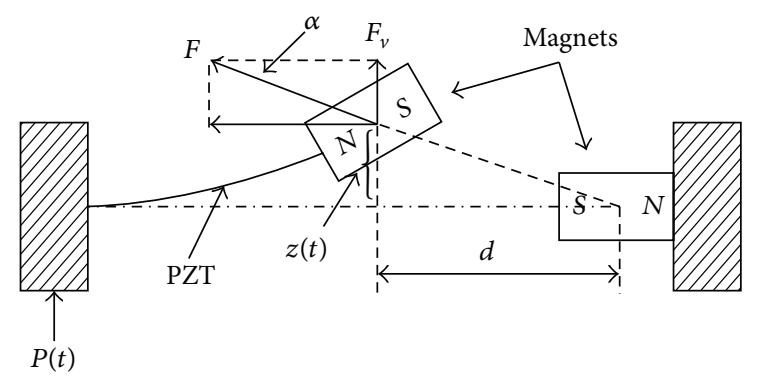

FIGURE 1: Schematic diagram of nonlinear PVEH system with two magnets.

based on which chaotic dynamics mechanisms of broadband PVEH enhanced by nonlinearity are explained. In Section 4, the Melnikov function of the nonlinear PVEH system is defined and calculated. Based on it, two necessary conditions of determining the distance are proposed. Simulations are done in Section 5 to validate the theoretic results. Finally, conclusions are discussed in Section 6.

\section{State-Space Model of One Nonlinear PVEH System with Nonlinear Magnetic Force}

As shown in Figure 1, a typical nonlinear piezoelectric energy harvesting system is composed of a piezoelectric cantilever beam and a pair of permanent magnets. The first magnet is mounted on the free end of the beam as a proof mass and the second magnet is oriented with opposite polarity to the first magnet. The distance between the two magnets is denoted as $d$. The length, width, and thickness of the piezoelectric beam are denoted as $l, w$, and $h$, respectively. The proof mass is assumed to be a lumped mass $M$. The vibration displacement of the mass at time $t$ is denoted as $z(t)$.

According to previous conclusions [7, 14], electromechanical coupling model of the nonlinear PVEH system in Figure 1 can be formulated as

$$
\begin{aligned}
& M_{\mathrm{eq}} \ddot{z}(t)+c_{\mathrm{eq}} \dot{z}(t)+K_{\mathrm{eq}} z(t)+\eta v_{p}(t)=P(t)+F_{v}(t), \\
& \eta \dot{z}(t)=C_{p} \dot{v}_{p}(t)+\frac{v_{p}(t)}{R_{L}},
\end{aligned}
$$

where $M_{\text {eq }}$ is the equivalent mass of the piezoelectric beam and the proof mass. $c_{\mathrm{eq}}$ is the equivalent damper of the piezoelectric beam. $K_{\mathrm{eq}}$ is the equivalent stiffness of the piezoelectric beam. $\eta$ is the piezoelectric coupling coefficient. $C_{p}$ is the equivalent capacitor of the piezoelectric beam. $z(t)$ is the displacement of $M_{\mathrm{eq}} \cdot R_{L}$ is the purely resistive load. $P(t)$ is the force applied to the mass of the equivalent SDOF model. $d$ is the horizontal distance of two magnets. $F_{v}(t)$ is the vertical component of the repulsive magnetic force $(F(d))$. Generally speaking, $z(t)$ is much smaller than $d$, so that we will have

$$
F_{v}(t)=\frac{F(d)}{d} z(t)-\frac{F(d)}{2 d^{3}} z^{3}(t) .
$$


Furthermore, the repulsive magnetic force, $F(d)$, can be approximated as [20]

$$
F(d)=W H t_{m}^{1 / 3} B_{r}|B(d)| f(d),
$$

where $W, H$, and $t_{m}$ correspond to the width, height, and thickness of the magnet, respectively. $B_{r}$ is the residual flux density of the magnet. $B(d)$ is calculated by [20]

$$
\begin{aligned}
& B(d)=\frac{B_{r}}{\pi}\left(\tan ^{-1} \frac{W H}{2 d \sqrt{W^{2}+H^{2}+4 d^{2}}}\right. \\
& \left.-\tan ^{-1} \frac{W H}{2(l+d) \sqrt{W^{2}+H^{2}+4(l+d)^{2}}}\right) .
\end{aligned}
$$

$f(d)$ is an experiential modification function, which is calculated by [20]

$$
f(d)=\left(1.749+1.145 e^{-d}\right) \times 10^{6} .
$$

According to the Melnikov theory, the first step is to rewrite (1) into a state-space form in order to use the Melnikov method to analyze nonlinear dynamic systems. For the sake of simplicity, $P(t)$ is assumed to be a harmonic excitation; that is, $P(t)=P_{m} \cos \omega t . P_{m}, \omega$ are the excitation magnitude and angular frequency, respectively. Then (1) can be transformed into

$$
\begin{aligned}
\ddot{z}(t) & +A_{1} \dot{z}(t)+A_{2} z(t) \\
= & \frac{A_{3} F(d)}{d} z(t)-\frac{A_{3} F(d)}{2 d^{3}} z^{3}(t)+A_{3} P_{m} \cos \omega t \\
& -A_{4} v_{p}(t), \\
\dot{v}_{p}(t) & =A_{5} v_{P}(t)+A_{6} \dot{z}(t),
\end{aligned}
$$

where $A_{1}=c_{\text {eq }} / M_{\text {eq }}, A_{2}=K_{\text {eq }} / M_{\text {eq }}, A_{3}=1 / M_{\text {eq }}, A_{4}=$ $\eta / M_{\mathrm{eq}}, A_{5}=-1 / R_{L} C_{p}$, and $A_{6}=\eta / C_{p}$.

By denoting $z(t) \triangleq z, v_{p}(t) \triangleq v_{p}, y(t)=\dot{z}(t) \triangleq y$, statespace model of the nonlinear PVEH system can be obtained as (7) from (6):

$$
\begin{aligned}
\dot{z}= & y, \\
\dot{y}= & {\left[\frac{A_{3} F(d)}{d}-A_{2}\right] z-\frac{A_{3} F(d)}{2 d^{3}} z^{3}-A_{1} y } \\
& +A_{3} P_{m} \cos \omega t-A_{4} v_{p}, \\
\dot{v}_{p}= & A_{5} v_{p}+A_{6} y .
\end{aligned}
$$

\section{Chaotic Dynamics Mechanisms of Broadband PVEH Enhanced by Nonlinearity}

In order to use the Melnikov method, the PVEH system should be represented as a perturbed state-space model. Here it can be easily seen that the perturbation is due to vibration excitation, damping, and electromechanical coupling. Then the perturbed state-space model can be represented as

$$
\begin{aligned}
\dot{z}= & y, \\
\dot{y}= & {\left[\frac{A_{3} F(d)}{d}-A_{2}\right] z-\frac{A_{3} F(d)}{2 d^{3}} z^{3} } \\
& +\varepsilon\left[A_{3} P_{m} \cos \omega t-A_{4} v_{p}-A_{1} y\right], \\
\dot{v}_{p}= & A_{5} v_{p}+A_{6} y,
\end{aligned}
$$

where $\varepsilon$ is a small factor that measures the smallness of the perturbation.

The classical Melnikov method is mainly used for twodimensional systems. However, (8) is obviously a threedimensional system, so here it is difficult to directly use the Melnikov method. In order to overcome this problem, one solution in [19] is referred to for converting the threedimensional system into a two-dimensional system. Then, (8) can be represented as a two-dimensional form as follows:

$$
\mathbf{X}=\mathbf{f}(\mathbf{X})+\varepsilon \mathbf{g}(\mathbf{X}, t)
$$

where

$$
\mathbf{X}=\left(\begin{array}{l}
z \\
y
\end{array}\right),
$$

$$
\begin{aligned}
\mathbf{f}(\mathbf{X}) & =\left(\begin{array}{c}
y \\
{\left[\frac{A_{3} F(d)}{d}-A_{2}\right] z-\frac{A_{3} F(d)}{2 d^{3}} z^{3}}
\end{array}\right), \\
\mathbf{g}(\mathbf{X}, t) & =\left(\begin{array}{c}
0 \\
A_{3} P_{m} \cos \omega t-A_{4} v_{p}-A_{1} y
\end{array}\right) .
\end{aligned}
$$

Obviously, when $\varepsilon=0$, the unperturbed system is a nonlinear planar dynamic system. Under this case, potential function of the unperturbed PVEH system can be calculated as follows:

$$
V(z)=-\frac{1}{2}\left[\frac{A_{3} F(d)}{d}-A_{2}\right] z^{2}+\frac{A_{3} F(d)}{8 d^{3}} z^{4} .
$$

It can be seen from (11) that potential function of the unperturbed PVEH system is closely related to the distance $(d)$. When the distance $(d)$ is properly selected, it will be similar to the curve in Figure 2. That is to say, it will be a double-well dynamic system including two potential barriers. Indeed, the bistability provides essential physical foundation of enhancing broadband PVEH, which has become a research hot spot in recent years [21-23].

According to (9), perturbed forces of the nonlinear dynamic system are from excitation, damping, and electromechanical coupling forces when $\varepsilon \neq 0$. Depending on the amount of $\varepsilon$, different vibration behaviors of the nonlinear $\mathrm{PVEH}$ system will occur. When the perturbed force is much 


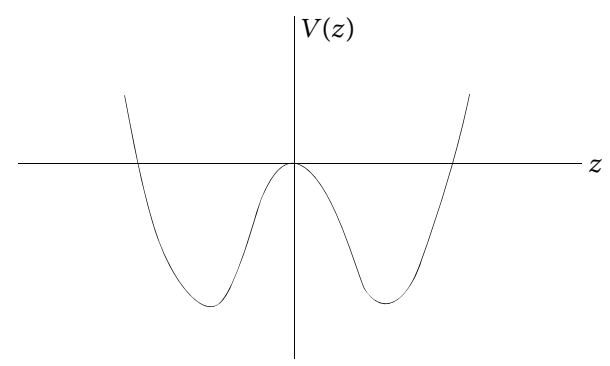

FIGURE 2: Potential function of the unperturbed nonlinear planar system.

smaller, it is difficult for the system to cross the potential barrier. Under this case, the system will periodically be around one of stable positions according to initial conditions. When the perturbed force is too large, the system is able to cross the potential barrier. Under this case, the system will periodically vibrate between two potential wells, leading to a large-amplitude motion. When the perturbed force is intermediate, the motion will be irregular. Under this case, the system may vibrate around one of stable positions or escape stochastically between two stable positions if initial values and excitation frequency satisfy necessary conditions. This kind of transitions is also called a chaotic motion, which is much expected for PVEH. The reason is that continuous transitions between two potential wells definitely provide much more power than chaotic motions, but it may not be guaranteed for broadbands, while chaotic motions at least can provide intermittent large-amplitude oscillations in a broadband. Thus one can understand why nonlinearity can be used to achieve high-efficiency broadband PVEH.

In practice, however, the key is how to determine a proper distance $(d)$ in order to make the nonlinear PVEH system generate a chaotic motion under a given vibration excitation. Fortunately, the Melnikov criterion helps in an indirect way in ascertaining the values of the different parameters for bifurcations. That is to say, when the Melnikov function has simple zeros independent of the small perturbation factor, the stable and unstable manifolds of the perturbed system will intersect each other transversely. Then it implies chaos-like behaviors may occur in the context of the Smale horseshoe map, which is also the research basis of this paper.

\section{Melnikov Function-Based Necessary Conditions of Broadband PVEH Using Nonlinearity}

As stated in Section 3, nonlinear magnetic forces can be used to cause the nonlinear PVEH system to be in chaotic conditions. In engineering applications, the distance $(d)$ between two magnets is the most key parameter once geometric and material properties of the harvesting structure are fixed. However, how to determine $d$ is always a difficult task. In order to solve this problem, necessary conditions of determining the distance are proposed based on the Melnikov function.
Based on (9), it can be easily seen that unperturbed nonlinear PVEH system is a Hamiltonian system and the Hamiltonian function is defined as follows:

$$
\begin{aligned}
H(y, z)= & \frac{1}{2} y^{2}-\frac{1}{2}\left[\frac{A_{3} F(d)}{d}-A_{2}\right] z^{2} \\
& +\frac{A_{3} F(d)}{8 d^{3}} z^{4}=\lambda,
\end{aligned}
$$

where $\lambda$ denotes one orbit in the phase plane of the Hamiltonian system, which depends on initial conditions.

Next three singular points of the unperturbed nonlinear PVEH system can be calculated as follows by setting $y=\dot{y}=$ 0 :

$$
\begin{aligned}
& Q_{0}=(0,0), \\
& Q_{1}=\left(d \sqrt{\frac{2\left(A_{3} F(d)-A_{2} d\right)}{A_{3} F(d)}}, 0\right), \\
& Q_{2}=\left(-d \sqrt{\frac{2\left(A_{3} F(d)-A_{2} d\right)}{A_{3} F(d)}}, 0\right) .
\end{aligned}
$$

Under this case, it can be seen from basic definitions that $Q_{0}$ is a saddle point and $Q_{1}, Q_{2}$ are two central points located at two sides of $Q_{0}$. However, it must be noted that $Q_{1}$ and $Q_{2}$ exist only when (14) is satisfied:

$$
\frac{F(d)}{d}>\frac{A_{2}}{A_{3}} .
$$

Thus (14) is the first necessary condition of determining the distance $(d)$.

Furthermore, the orbit described by (12) is a homoclinic orbit which will cross the saddle point $\left(Q_{0}\right)$. Under this case, $\lambda$ is equal to zero. Then we will have

$$
y= \pm z \sqrt{\alpha-\beta z^{2}}=\dot{z}
$$

where $\alpha=\left[A_{3} F(d)-d A_{2}\right] / d$ and $\beta=A_{3} F(d) / 4 d^{3}$. The sign " \pm " just represents the right and left parts of the orbit in the phase plane.

Due to its symmetry, only the sign " + " is considered here. Then (15) can be transformed into

$$
\int \frac{1}{z \sqrt{\alpha-\beta z^{2}}} d z=\int d t .
$$

By solving (16), analytic solutions of the homoclinic orbit can be obtained as

$$
\begin{aligned}
& z^{H}(t)=\sqrt{\frac{\alpha}{\beta}} \sec (i \sqrt{\alpha} t), \\
& y^{H}(t)=\frac{\alpha i}{\sqrt{\beta}} \sec (i \sqrt{\alpha} t) \tan (i \sqrt{\alpha} t),
\end{aligned}
$$

where the superscript " $H$ " denotes a homoclinic orbit and " $i$ " denotes an imaginary number. 
Substituting (17) into (8), the homoclinic orbit $\left(v_{p}^{H}(t)\right)$ of the output voltage is calculated as

$$
\begin{aligned}
& v_{p}^{H}(t) \\
& \quad=\frac{i \alpha A_{6}}{\sqrt{\beta}} e^{A_{5} t}\left[\int_{0}^{t} e^{-A_{5} t^{\prime}} \sec \left(i \sqrt{\alpha} t^{\prime}\right) \tan \left(i \sqrt{\alpha} t^{\prime}\right) d t^{\prime}\right] .
\end{aligned}
$$

Finally, the Melnikov function of the perturbed nonlinear PVEH system can be defined as follows:

$$
M\left(t_{0}\right)=\int_{-\infty}^{+\infty} \mathbf{f}\left[z^{H}, y^{H}\right] \wedge \mathbf{g}\left[z^{H}, y^{H}, t+t_{0}\right] d t
$$

where " $\wedge$ " denotes the wedge product.

By substituting (17) and (18) into (19), we will have

$$
M\left(t_{0}\right)=-\Omega_{1}-\Omega_{2}+\Omega_{3} \sin \omega t_{0} S(\omega)
$$

where $\Omega_{1}=2 A_{1} \alpha \sqrt{\alpha} / 3 \beta, \quad \Omega_{2}=$ $-\left(A_{4} A_{6} \alpha^{2} / \beta\right) \int_{-\infty}^{+\infty} h(t) \sec (i \sqrt{\alpha} t) \tan (i \sqrt{\alpha} t) d t$, $\Omega_{3}=A_{3} P_{m}, S(\omega)=(\pi \omega / \sqrt{\beta}) \operatorname{sech}(\pi \omega / 2 \sqrt{\alpha})$, and $h(t)=e^{A_{5} t}\left[\int_{0}^{t} e^{-A_{5} t^{\prime}} \sec \left(i \sqrt{\alpha} t^{\prime}\right) \tan \left(i \sqrt{\alpha} t^{\prime}\right) d t^{\prime}\right]$. Here, $\Omega_{2}$ can be calculated numerically.

According to the Melnikov criteria, homoclinic bifurcation and chaotic motions may occur if $M\left(t_{0}\right)$ has simple zeros independent of $\varepsilon$. Then the following necessary condition must be satisfied based on (20):

$$
0<M_{0}=\left|\frac{\Omega_{1}+\Omega_{2}}{\Omega_{3} S(\omega)}\right|<1
$$

$\Omega_{1}, \Omega_{2}$ are closely related to $d$, so (21) is the second necessary condition of determining $d$.

Up to now, the proposed Melnikov necessary conditions of determining the distance are composed of (14) and (21). At the same time, one can see that the second necessary condition is also closely related to excitation amplitude $\left(P_{m}\right)$ and angular frequency $(\omega)$. Then one can expect that the excitation angular frequency $(\omega)$ in a wide band may satisfy the Melnikov conditions under a proper distance. By this way, one can understand why nonlinearity can be used to enlarge resonant bandwidth of one PVEH system.

\section{Simulation Validations}

In order to validate the above theoretical results, simulations are carried out to analyze the effects of key parameters on the Melnikov necessary conditions. Also output response characteristics of the nonlinear PVEH system are investigated under different vibration excitations. Firstly, geometric and material properties of the nonlinear piezoelectric structure are listed in Table 1. Under this case, resonant frequency of the piezoelectric cantilever beam is calculated as about $26.4 \mathrm{~Hz}$ without considering the second magnet. The excitation frequency is denoted as $f$. That is to say, $\omega=2 \pi f$. The load is fixed as $R_{L}=200 \mathrm{k} \Omega$.
TABLE 1: Geometric and material properties of the nonlinear piezoelectric structure.

\begin{tabular}{llc}
\hline Structure & Properties & Value \\
\hline \multirow{2}{*}{ Elastic base } & Density, $\rho_{s}\left(\mathrm{~kg} / \mathrm{m}^{3}\right)$ & 8500 \\
& Young's modulus, $E(\mathrm{Gpa})$ & 90 \\
\hline \multirow{3}{*}{ PZT-5H } & Thickness, $h_{p}(\mathrm{~mm})$ & 0.2 \\
& Density, $\rho_{s}\left(\mathrm{~kg} / \mathrm{m}^{3}\right)$ & 7500 \\
& Young's modulus, $E_{p}(\mathrm{Gpa})$ & 66 \\
& Piezoelectric constant, $d_{31}(\mathrm{pm} / \mathrm{V})$ & -190 \\
& Permittivity, $\varepsilon_{33}^{s} / \mathrm{nF} / \mathrm{m}$ & 15.93 \\
\hline \multirow{3}{*}{ Magnet } & Length, $L(\mathrm{~mm})$ & 12 \\
& Width, $W(\mathrm{~mm})$ & 6 \\
& Thickness, $H(\mathrm{~mm})$ & 7 \\
& Residual flux density, $B_{r}(\mathrm{~T})$ & 1.25 \\
\hline
\end{tabular}

5.1. Potential Function of the Unperturbed PVEH System. As stated in Section 3, potential function of the unperturbed PVEH system is an important physical basis of achieving broad bandwidth. Based on (11), potential functions under different distances are plotted and compared in Figure 3. One can see that the double well in the potential function will disappear once the distance is too large or small. The reason may be that when $d$ is very small, the magnetic force is so large that the system will be a monostable system. Under this case, neither a large-amplitude nor a chaotic motion will occur despite the vibration excitation, which is not useful for broadband PVEH. Again, one can understand why the distance $(d)$ should be determined carefully.

5.2. The Effects of the Excitation Amplitude on the Melnikov Necessary Conditions. As stated in Section 4, the first Melnikov necessary condition as (14) must be satisfied in order to cause homoclinic bifurcation and chaotic motion. Based on (14), it can be easily found that the distance should satisfy $d<9.5 \mathrm{~mm}$, which is independent of vibration excitations. Furthermore, the second Melnikov necessary condition as (21) is closely related to the excitation amplitude $\left(P_{m}\right)$, so we will focus on the effects of $P_{m}$ on $M_{0}$.

5.2.1. The Effects of $P_{m}$ on the Bandwidth. Here the distance is fixed as $d=6 \mathrm{~mm}$ according to the first Melnikov necessary condition. Then different excitation amplitudes are selected as $P_{m}=0.2,0.4,0.6,0.8$. For each $P_{m}$, frequency response of $M_{0}$ is calculated numerically as Figure 4, where the red line denotes the threshold. The following can be seen. (i) For any $d$ satisfying (14), there will be a broad frequency band at which (21) is also satisfied. That is to say, homoclinic bifurcation and chaotic motion may occur in the broadband frequency band. At the same time, the frequency band is always located at lowfrequency regions. (ii) As $P_{m}$ increases, the bandwidth will be enlarged and the central frequency will also increase. These results testify that broadband PVEH can be achieved by using proper nonlinearity.

5.2.2. The Effects of $P_{m}$ on the Distance. Here the excitation frequency is fixed as $f=20 \mathrm{~Hz}$. Then different excitation 


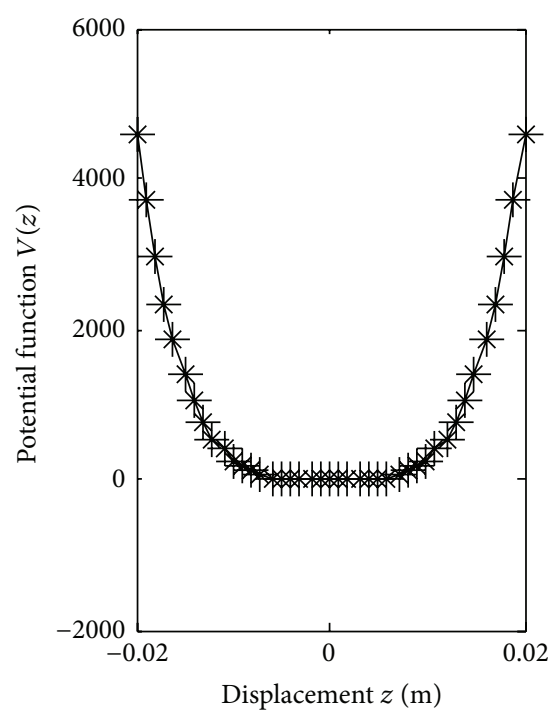

(a) $d=2 \mathrm{~mm}$

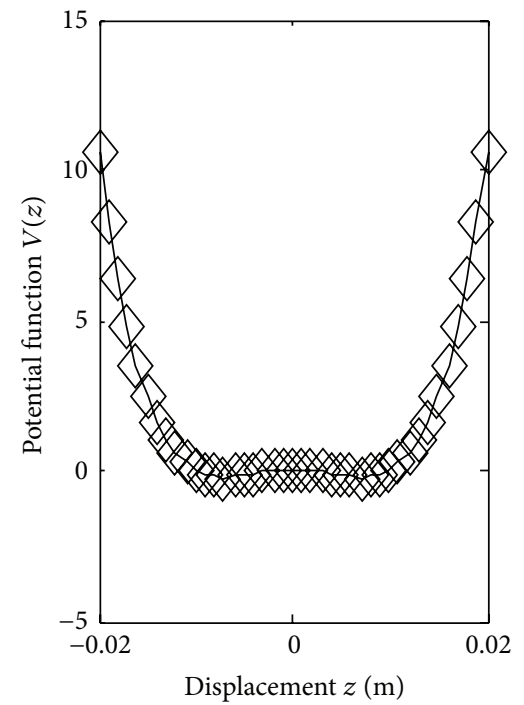

(d) $d=8 \mathrm{~mm}$

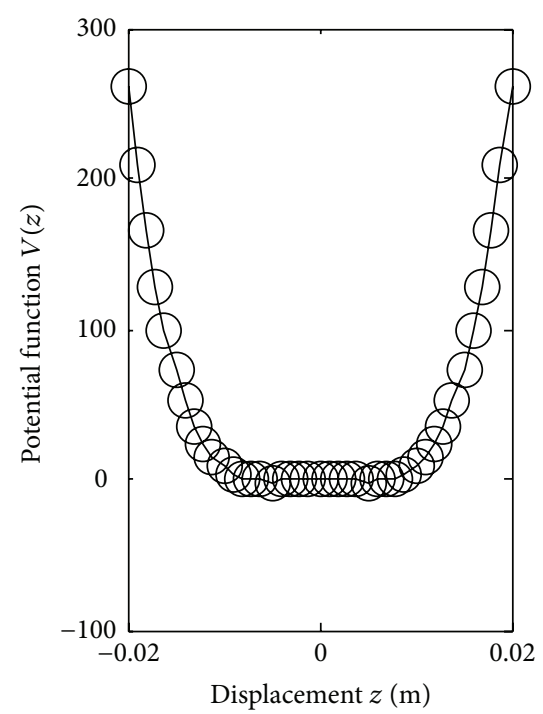

(b) $d=4 \mathrm{~mm}$

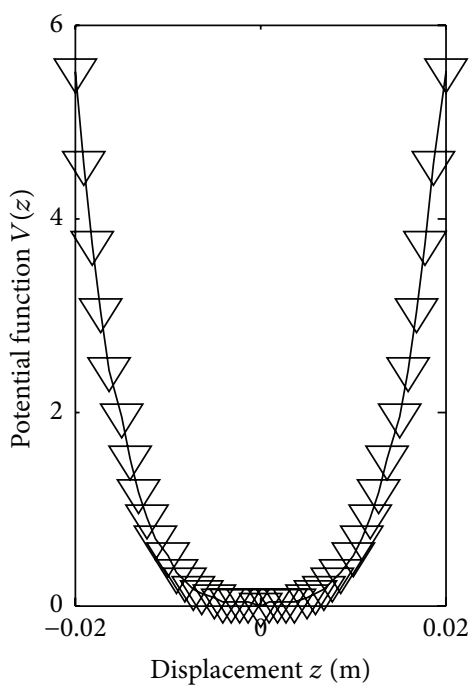

(e) $d=10 \mathrm{~mm}$

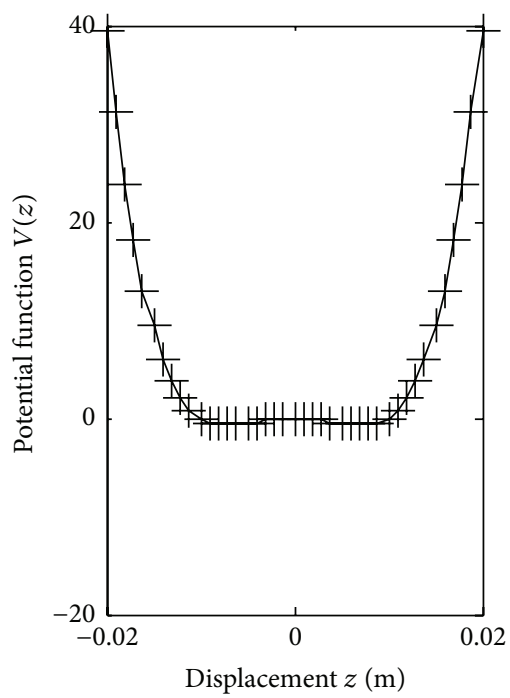

(c) $d=6 \mathrm{~mm}$

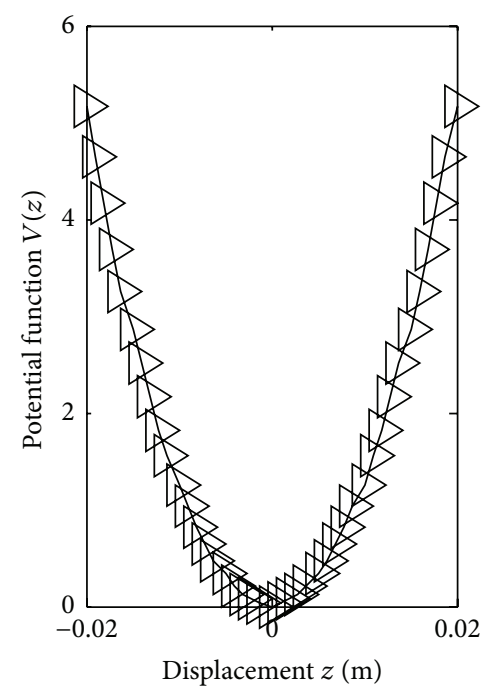

(f) $d=20 \mathrm{~mm}$

FIGURE 3: Potential functions of the unperturbed nonlinear PVEH system under different distances.

amplitudes are selected as $P_{m}=0.02,0.04,0.06,0.08$. For each $P_{m}$, the curves of $M_{0}$ with $d$ are calculated numerically as Figure 5, where the red line denotes the threshold. The following can be seen. (i) For any given $P_{m}$, there is a range of $d$ so that the two Melnikov necessary conditions are satisfied, which can help to design proper PVEH devices in practice. (ii) As $P_{m}$ increases, the range of $d$ will be extended.

\subsection{Melnikov Scale Factor $(S(f))$. Denoting $S(f) \triangleq$} $\left.S(\omega)\right|_{\omega=2 \pi f}$ in (20), it is obvious that whether the second Melnikov necessary condition is easily satisfied or not depends on $S(f)$ once $d$ and $P_{m}$ are given. Thus here $S(f)$ is called the Melnikov scale factor. Then the curves of $S(f)$ under different distances are shown in Figure 6. One can see the following. (i) Under a given $d$, there is an optimal excitation frequency at which $S(f)$ has a maximum value. That is to say, it is the most easy case to satisfy the second Melnikov necessary condition and generate chaotic motions. (ii) As $d$ increase, the optimal excitation frequency will shift to low-frequency regions.

5.4. Output Responses of the Nonlinear PVEH System under a Single-Frequency Excitation. In order to testify three types of motions of the nonlinear PVEH system under different excitation frequencies, three kinds of single-frequency excitations ( $f=5 \mathrm{~Hz}, f=12 \mathrm{~Hz}$, and $f=21 \mathrm{~Hz}$ ) are used, respectively. Here we choose $d=6 \mathrm{~mm}$ and $P_{m}=0.4$; then output responses of the nonlinear PVEH system are shown in Figures 7-9, including the phase plane, voltage, and displacement. 


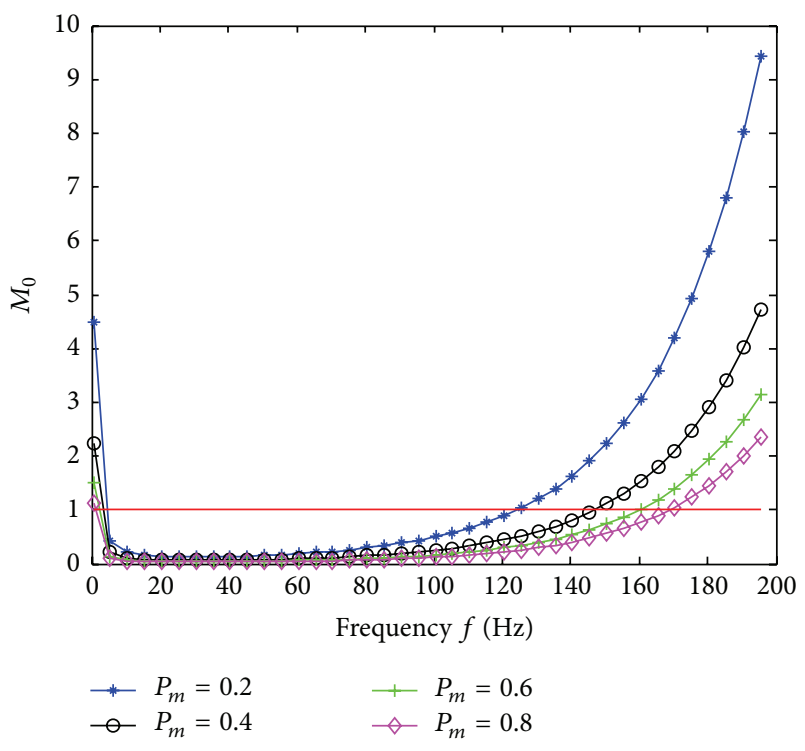

FIGURE 4: Frequency responses of $M_{0}$ under different excitation amplitudes.

It can be seen from the phase plane in Figure 7 that the nonlinear system vibrates periodically around one of the stable positions when $f=5 \mathrm{~Hz}$. Under this case, the stable vibration amplitude is small, leading to small output voltages. It can be seen from the phase plane in Figure 8 that the nonlinear system vibrates periodically between two stable points and a large-amplitude motion occurs when $f=12 \mathrm{~Hz}$. Under this case, the stable vibration amplitude is large, leading to large output voltages. It can be seen from the phase plane in Figure 9 that the nonlinear system escapes stochastically between two stable positions when $f=25 \mathrm{~Hz}$ and a chaotic motion occurs. Under this case, the stable vibration amplitude is also large.

Furthermore, average output powers under the above three motions are calculated as $4.5 \mathrm{~mW}, 177.4 \mathrm{~mW}$, and $87.2 \mathrm{~mW}$, respectively. It is obvious that average output power under a large-amplitude or chaotic motion is larger than that under a small-amplitude motion. Although average output power under a large-amplitude motion is the largest, it is much more sensitive to the excitation frequency. Thus it is difficult to keep large-amplitude motions due to interrupts in engineering applications. A chaotic motion can occur in a broad frequency band, so it is the most suitable for engineering applications.

Next, we choose $d=20 \mathrm{~mm}$ and $f=21 \mathrm{~Hz}$. Obviously, the first Melnikov necessary condition as (14) is not satisfied under this distance. At this time, the effect of the nonlinear magnetic force is very small and the nonlinear system degrades to a linear one. Then the phase plane, voltage, and displacement are also calculated as Figure 10. One can see the nonlinear system vibrates periodically around the equilibrium position, instead of a chaotic motion. Under this case, both the stable vibration amplitude and output voltages are smaller than the counterparts under a chaotic motion. Its average output power is calculated as $24.5 \mathrm{~mW}$. By comparing

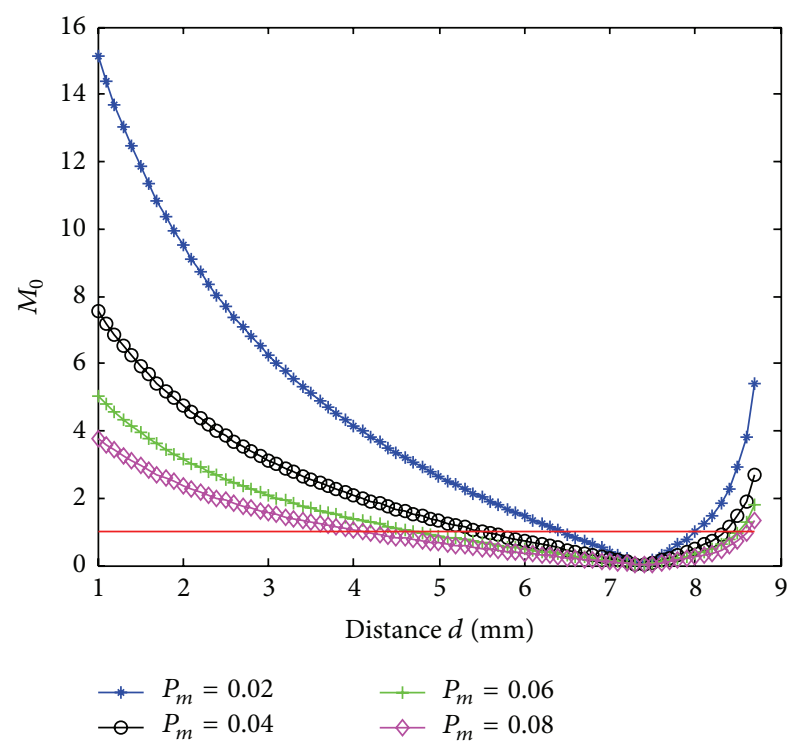

FIgURE 5: The curves of $M_{0}$ with $d$ under different excitation amplitudes.

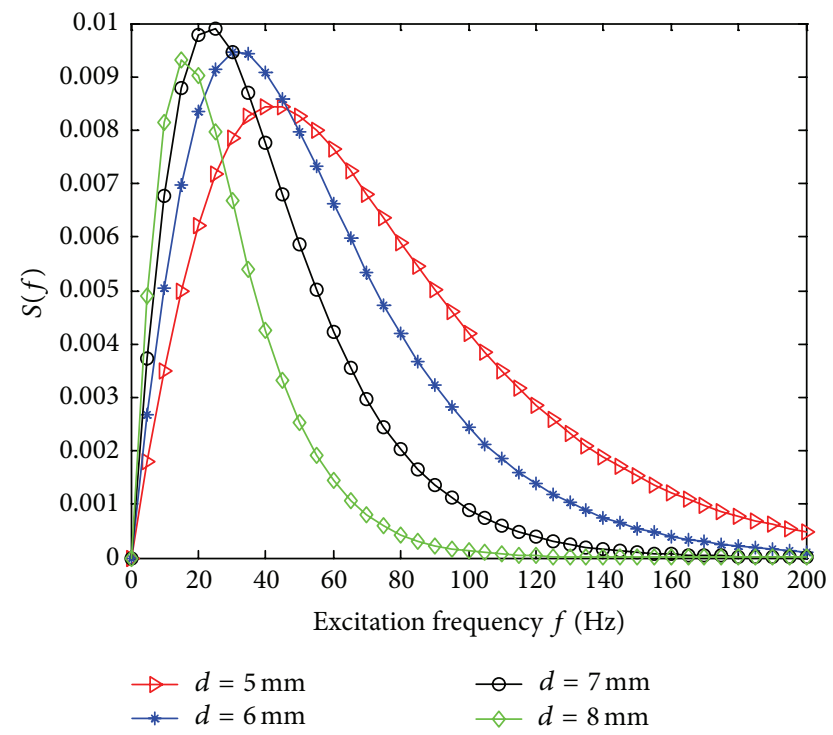

FIgURE 6: The curves of $S(f)$ under different distances.

Figure 10 with Figure 7, one can see that a nonlinear PVEH system with a too small distance is even worse than a linear PVEH system.

5.5. Output Responses of the Nonlinear PVEH System under a Broadband Excitation. In order to validate broadband characteristics of the nonlinear PVEH system, a time-varying excitation frequency is used here; that is, $f=10 t, t \in[0,10]$. Two distances, $d=6 \mathrm{~mm}$ and $d=20 \mathrm{~mm}$, are chosen for comparisons. Obviously, the former denotes a nonlinear PVEH system and the latter denotes a linear one. Then output voltages of the two PVEH systems are calculated as Figure 11. It can be seen that the bandwidth of a nonlinear PVEH system is larger than that of a linear one, while the maximum output 

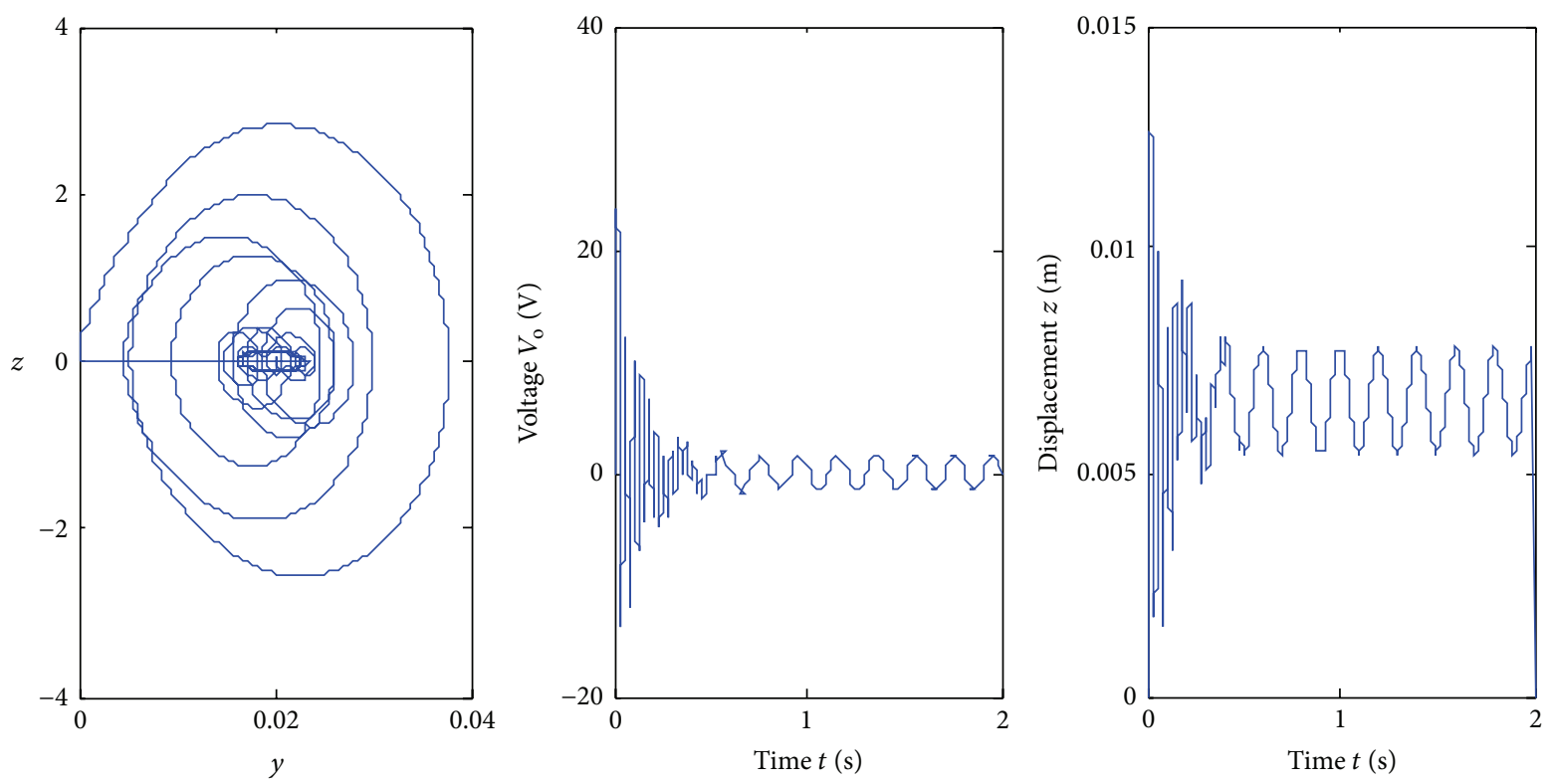

Figure 7: A periodic motion around a stable position when $f=5 \mathrm{~Hz}$.
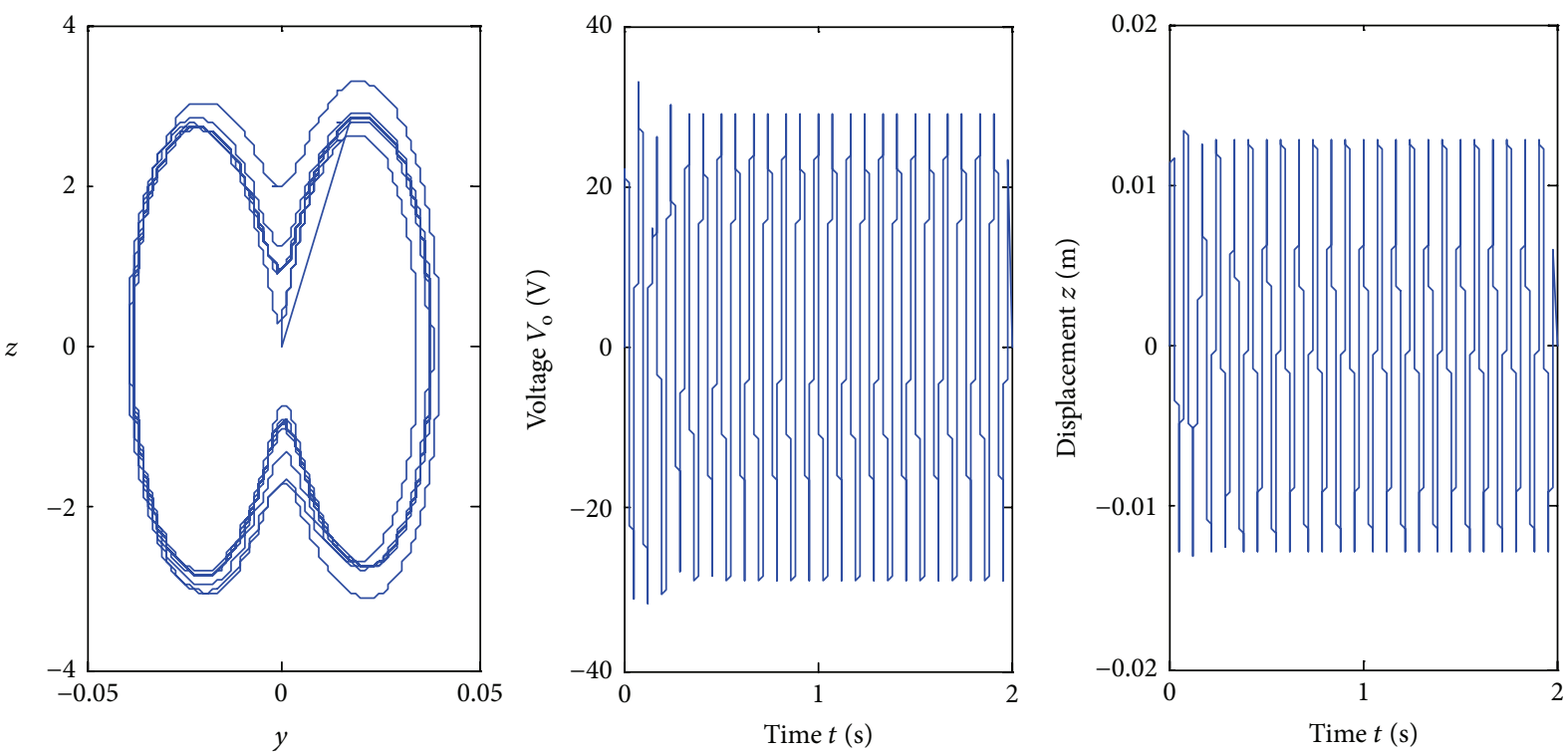

Figure 8: A large-amplitude motion across two stable positions when $f=12 \mathrm{~Hz}$.

voltage of a linear system is only achieved near its resonant frequency.

\section{Conclusions}

It has been pointed out that nonlinearity by magnetic force can be used to extend resonant frequency bandwidth of a PVEH system. However, how to determine the distance between two magnets is a key problem in engineering applications. In this paper, the Melnikov theory is introduced to solve it. Firstly, the Melnikov state-space model of the nonlinear PVEH system is built. Chaotic dynamics mechanisms of achieving broadband PVEH are exposed by the potential function of the unperturbed nonlinear PVEH system. Then the Melnikov function of the nonlinear PVEH system is defined, based on which two Melnikov necessary conditions of determining the distance are obtained. Finally, simulations are done to testify the theoretic results. Main conclusions include the following. (i) Both a too large and a small distance are not helpful to form double wells. The distance should satisfy the first Melnikov necessary condition. (ii) For a given distance satisfying (14), there will 

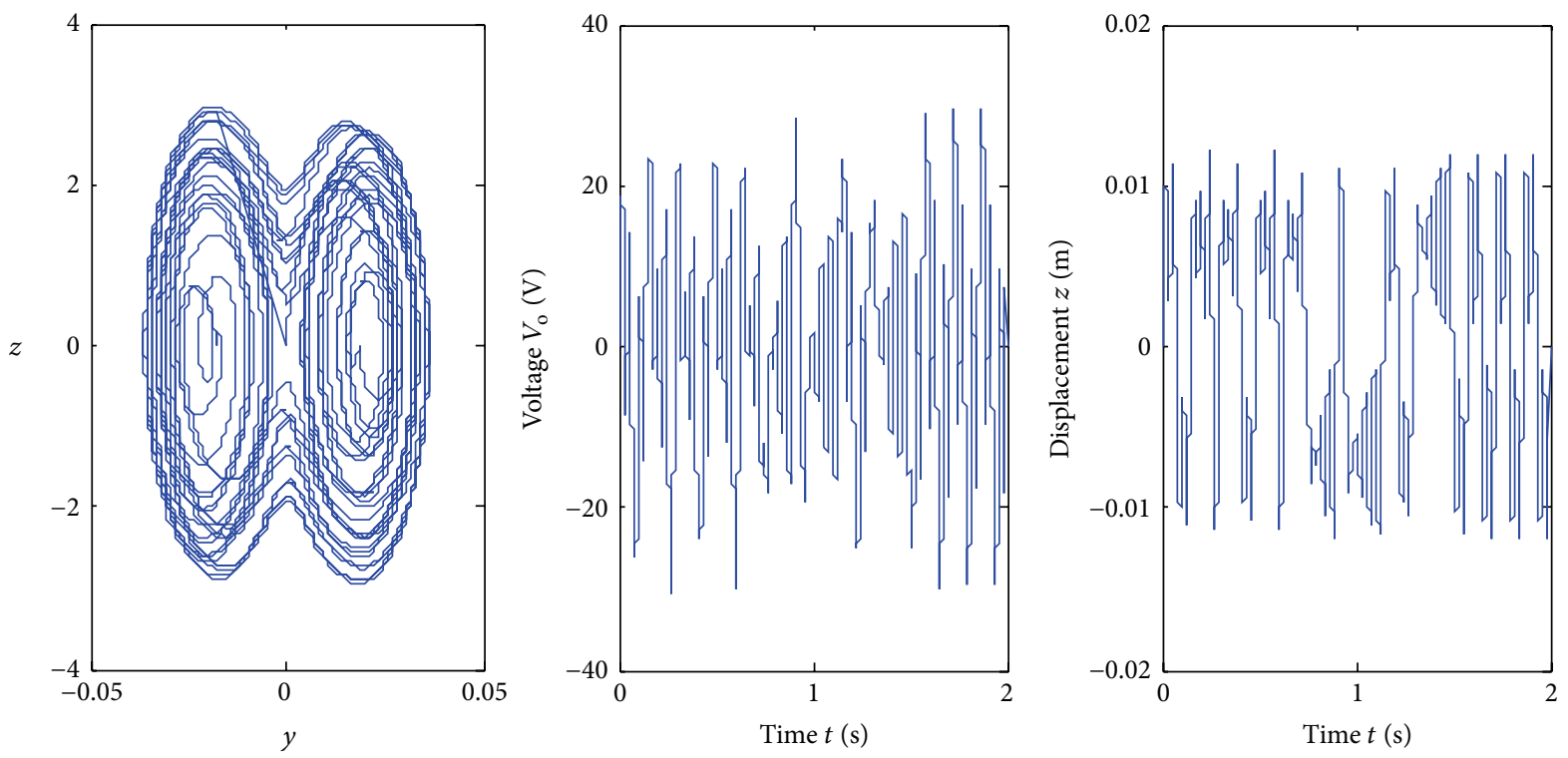

FIgURE 9: A homoclinic bifurcation and chaotic motion when $f=21 \mathrm{~Hz}$.
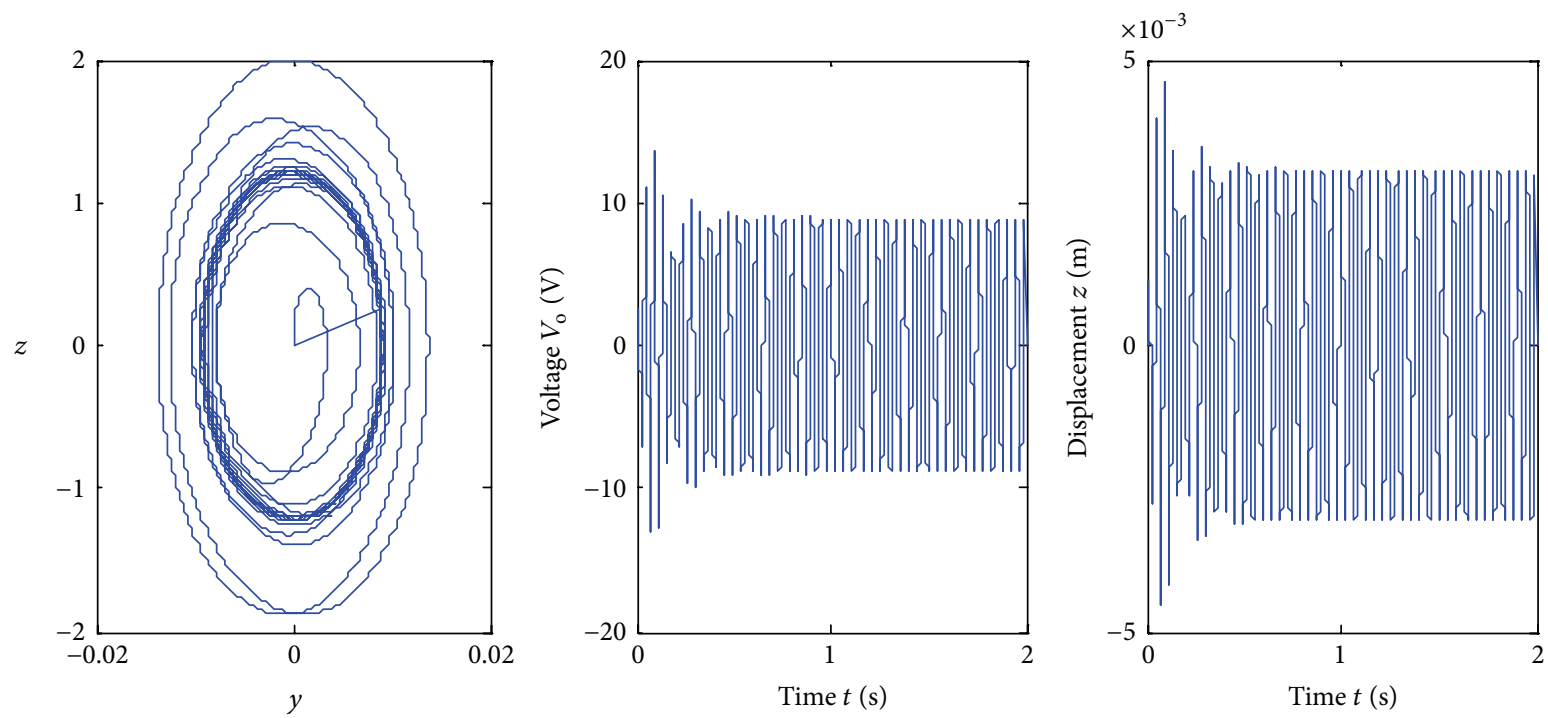

FIGURE 10: A periodic motion around the equilibrium position when $d=20 \mathrm{~mm}$ and $f=21 \mathrm{~Hz}$.

be a broad frequency band satisfying the second Melnikov necessary condition. For a given excitation frequency, there also will be a range of the distance satisfying the two Melnikov necessary conditions. (iii) For any given distance satisfying the first Melnikov necessary condition, there will be an excitation frequency at which the second Melnikov necessary condition is the most easily satisfied. (iv) Under a single-frequency excitation, the nonlinear PVEH system can generate a periodic vibration around a stable point, a largeamplitude vibration around two stable points, or a chaotic vibration. In addition, it must be noted that the proposed Melnikov conditions are just necessary, not sufficient. The problem needs to be investigated further in future works.
Despite it, however, the above conclusions will still be much more significant for designing nonlinear PVEH devices in practice.

\section{Conflict of Interests}

The authors declare that there is no conflict of interests regarding the publication of this paper.

\section{Acknowledgment}

This work was supported by the National Natural Science Foundation of China (Grants nos. 51275520 and 51577189). 


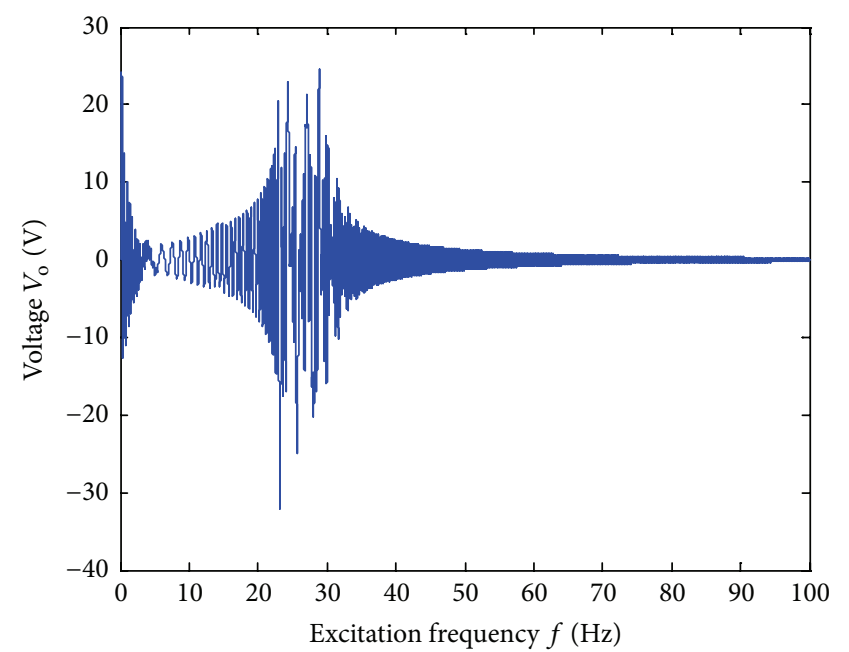

(a) $d=6 \mathrm{~mm}$

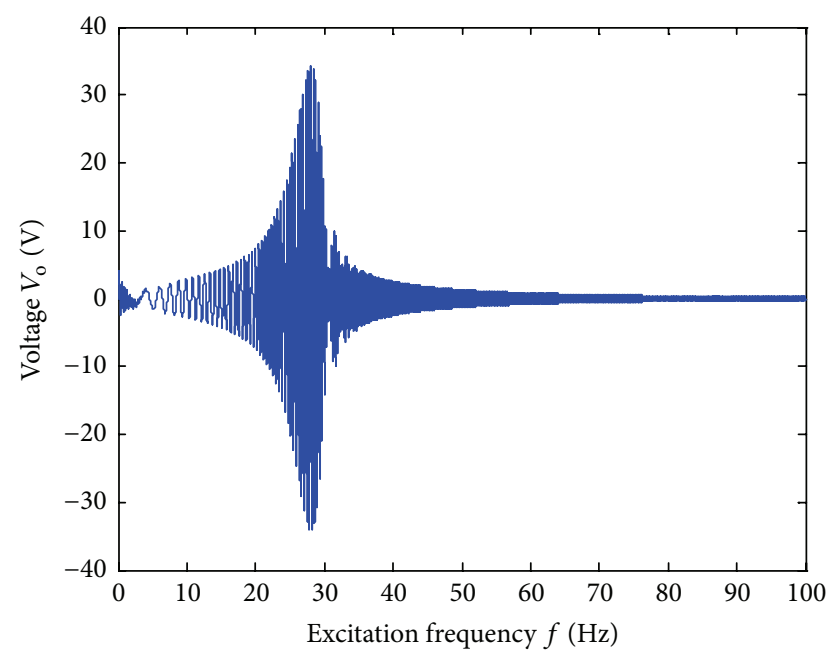

(b) $d=20 \mathrm{~mm}$

FIGURE 11: Output voltages of the nonlinear PVEH system under a broadband excitation.

\section{References}

[1] L. G. W. Tvedt, D. S. Nguyen, and E. Halvorsen, "Nonlinear behavior of an electrostatic energy harvester under wide and narrowband excitation," Journal of Microelectromechanical Systems, vol. 19, no. 2, Article ID 5404427, pp. 305-316, 2010.

[2] A. Rahimi, O. Zorlu, A. Muhtaroglu, and H. Kulah, "Fully selfpowered electromagnetic energy harvesting system with highly efficient dual rail output," IEEE Sensors Journal, vol. 12, no. 6, pp. 2287-2298, 2012.

[3] K. Junwu, Q. Jinhao, T. Kehong, Z. Kongjun, and S. Chenghui, "Modeling and simulation of piezoelectric composite diaphragms for energy harvesting," International Journal of Applied Electromagnetics and Mechanics, vol. 30, no. 1-2, pp. 95-106, 2009.

[4] S. Roundy, "On the effectiveness of vibration-based energy harvesting," Journal of Intelligent Material Systems and Structures, vol. 16, no. 10, pp. 809-823, 2005.

[5] L. Gammaitoni, I. Neri, and H. Vocca, "Nonlinear oscillators for vibration energy harvesting," Applied Physics Letters, vol. 94, no. 16, Article ID 164102, 2009.

[6] Z. S. Chen, B. Guo, Y. T. Luo, and Y. M. Yang, "Numerical investigations into the effects of multiple parameters on nonlinear piezoelectric vibration energy harvesters," Advances in Mechanical Engineering, vol. 6, Article ID 604704, 2014.

[7] S. C. Stanton, C. C. McGehee, and B. P. Mann, "Nonlinear dynamics for broadband energy harvesting: investigation of a bistable piezoelectric inertial generator," Physica D: Nonlinear Phenomena, vol. 239, no. 10, pp. 640-653, 2010.

[8] H. Vocca, I. Neri, F. Travasso, and L. Gammaitoni, "Kinetic energy harvesting with bistable oscillators," Applied Energy, vol. 97, no. 9, pp. 771-776, 2012.

[9] C. R. McInnes, D. G. Gorman, and M. P. Cartmell, "Enhanced vibrational energy harvesting using nonlinear stochastic resonance," Journal of Sound and Vibration, vol. 318, no. 4-5, pp. 655$662,2008$.

[10] F. Cottone, H. Vocca, and L. Gammaitoni, "Nonlinear energy harvesting," Physical Review Letters, vol. 102, no. 8, Article ID 080601, 2009.
[11] D. A. W. Barton, S. G. Burrow, and L. R. Clare, "Energy harvesting from vibrations with a nonlinear oscillator," Journal of Vibration and Acoustics, vol. 132, no. 2, Article ID 021009, 2010.

[12] W. Q. Liu, A. Badel, and F. Formosa, "A wideband integrated piezoelectric bistable generator: experimental performance evaluation and potential for real environmental vibrations," Journal of Intelligent Material Systems and Structures, vol. 26, no. 7, pp. 872-877, 2014.

[13] X. Xing, J. Lou, G. M. Yang, O. Obi, C. Driscoll, and N. X. Sun, "Wideband vibration energy harvester with high permeability magnetic material," Applied Physics Letters, vol. 95, no. 13, Article ID 134103, 2009.

[14] Z.-S. Chen and Y.-M. Yang, "Stochastic resonance mechanism for wideband and low frequency vibration energy harvesting based on piezoelectric cantilever beams," Acta Physica Sinica, vol. 60, no. 7, Article ID 074301, 2011.

[15] B. P. Mann and N. D. Sims, "Energy harvesting from the nonlinear oscillations of magnetic levitation," Journal of Sound and Vibration, vol. 319, no. 1-2, pp. 515-530, 2009.

[16] L. Tang, Y. Yang, and C.-K. Soh, "Improving functionality of vibration energy harvesters using magnets," Journal of Intelligent Material Systems and Structures, vol. 23, no. 13, pp. 1433-1449, 2012.

[17] L. Tang and Y. Yang, "A nonlinear piezoelectric energy harvester with magnetic oscillator," Applied Physics Letters, vol. 101, no. 9, Article ID 094102, 2012.

[18] K. Yagasaki, "Chaos in a pendulum with feedback control," Nonlinear Dynamics, vol. 6, no. 2, pp. 125-142, 1994.

[19] S. C. Stanton, B. P. Mann, and B. A. M. Owens, "Melnikov theoretic methods for characterizing the dynamics of the bistable piezoelectric inertial generator in complex spectral environments," Physica D: Nonlinear Phenomena, vol. 241, no. 6, pp. 711$720,2012$.

[20] X. D. Xie, Q. Wang, and N. Wu, "A ring piezoelectric energy harvester excited by magnetic forces," International Journal of Engineering Science, vol. 77, pp. 71-78, 2014.

[21] R. L. Harne and K. W. Wang, "A review of the recent research on vibration energy harvesting via bistable systems," Smart Materials and Structures, vol. 22, no. 2, Article ID 023001, 2013. 
[22] L. Tang, Y. Yang, and C. K. Soh, "Broadband vibration energy harvesting techniques," in Advances in Energy Harvesting Methods, pp. 17-61, Springer, New York, NY, USA, 2013.

[23] S. P. Pellegrini, N. Tolou, M. Schenk, and J. L. Herder, "Bistable vibration energy harvesters: a review," Journal of Intelligent Material Systems and Structures, vol. 24, no. 11, pp. 1303-1312, 2013. 


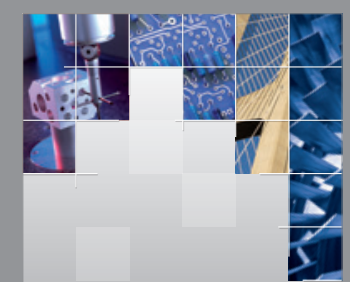

\section{Enfincering}
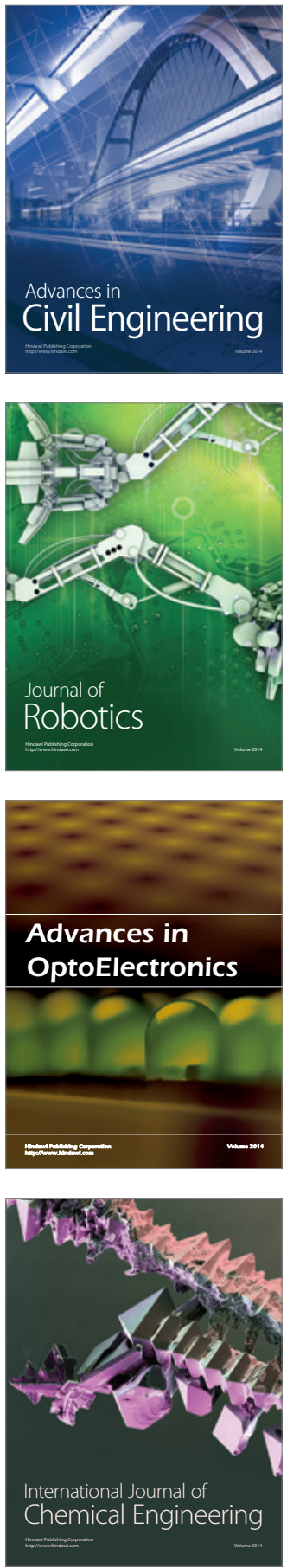

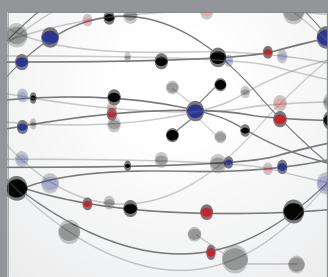

The Scientific World Journal

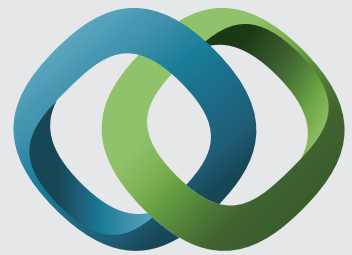

\section{Hindawi}

Submit your manuscripts at

http://www.hindawi.com
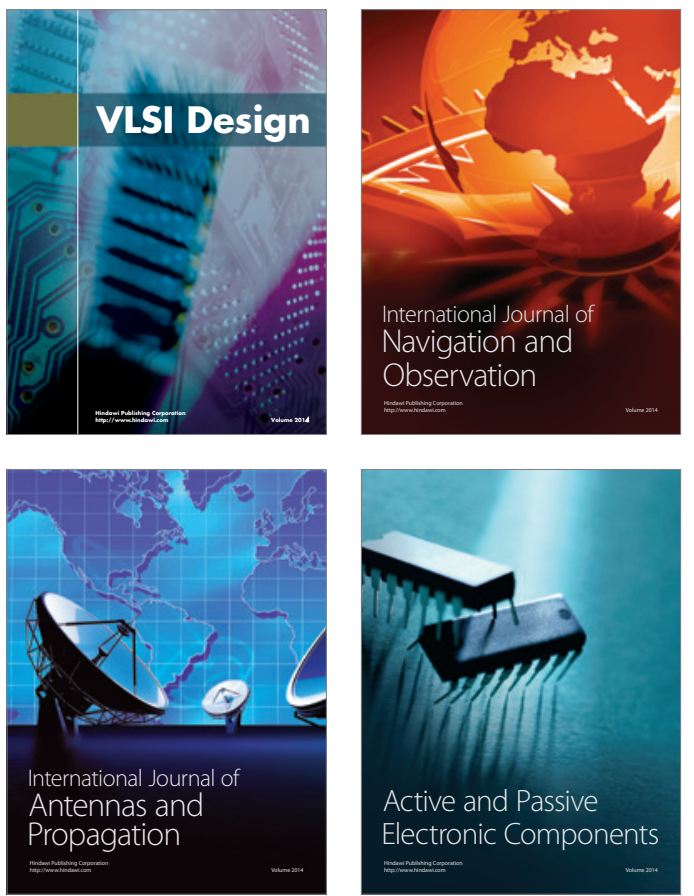
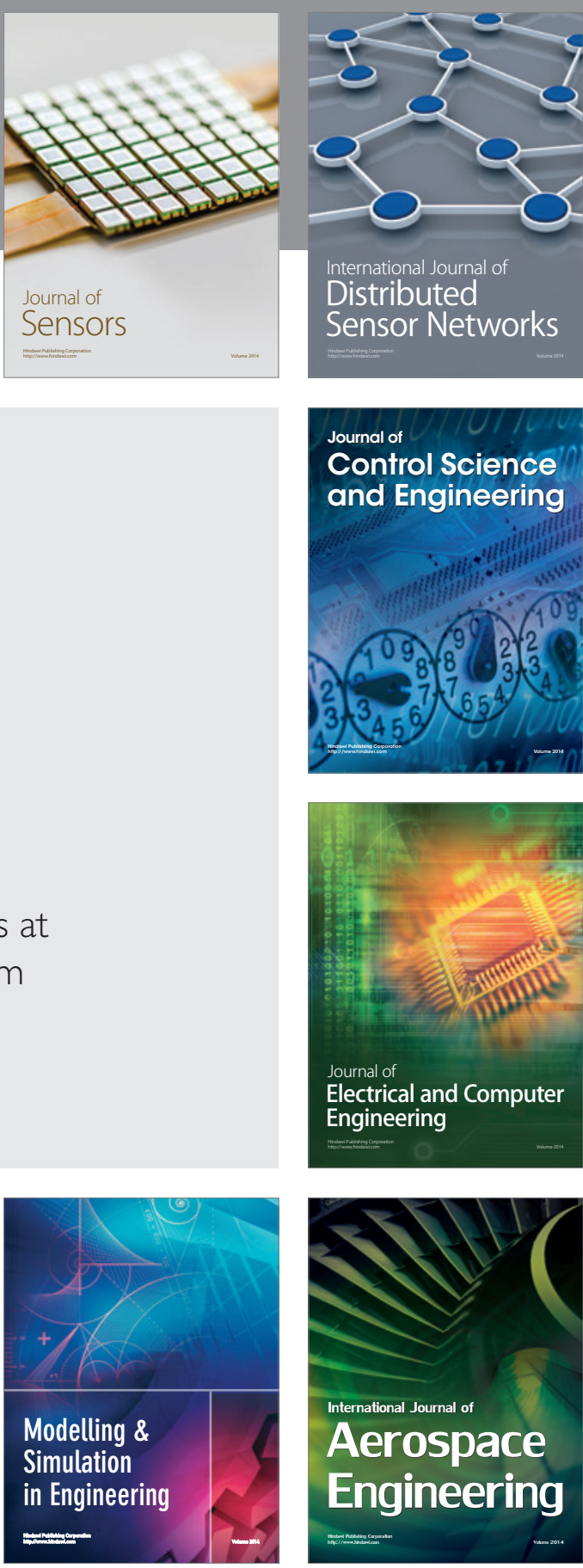

International Journal of

Distributed

Sensor Networks

Journal of

Control Science

and Engineering
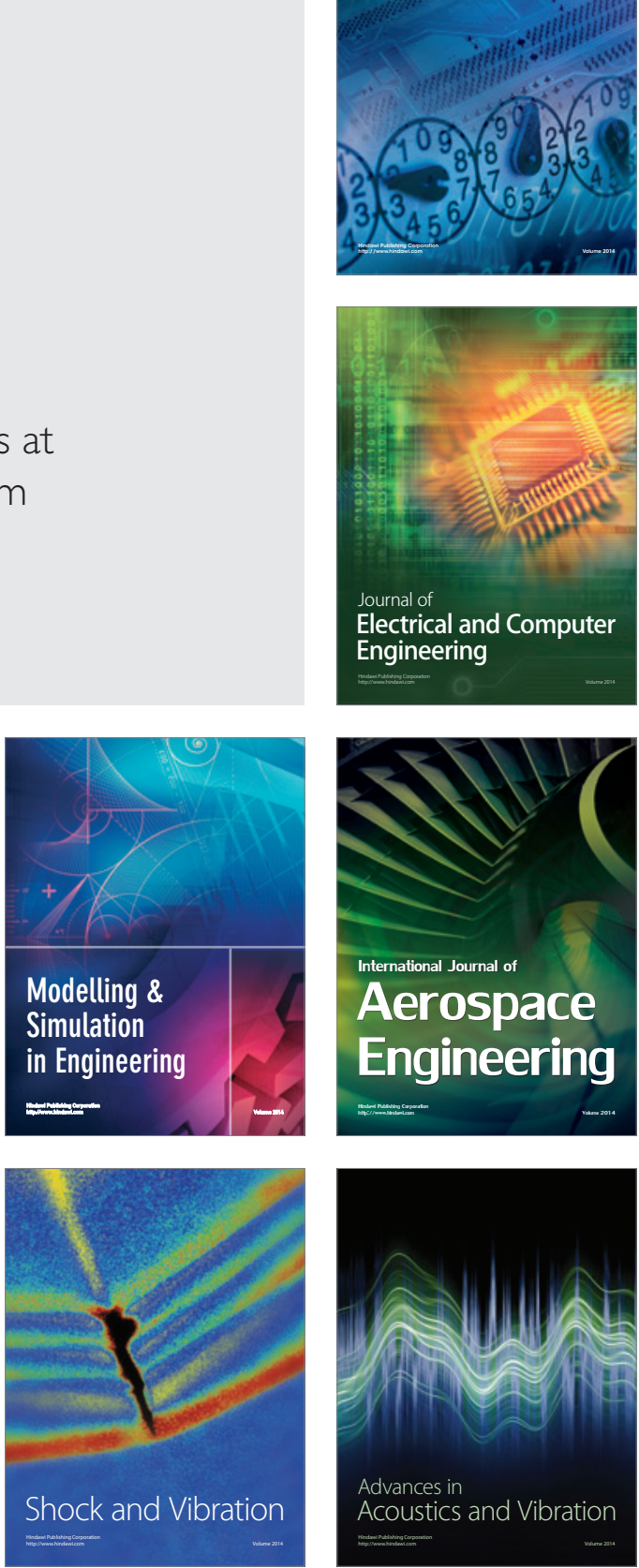